\title{
Precipitación de fierro y manganeso secuencial de efluentes de drenaje ácidos
}

\author{
Precipitation of fierro and sequential manganese of drain effluents
}

\author{
Julia Marilú Calderón de Alvarado ${ }^{1}$, María Carmen Tuiro Salvador ${ }^{2}$
}

Recibido: 10/06/2019 - Aprobado: 19/06/2020 - Publicado: 18/06/2021

\begin{abstract}
RESUMEN
Los efluentes ácidos de la lixiviación de los minerales sulfurados podrían ser un pasivo que retornara parte de su costo de tratamiento al ser obtenidos los elementos económicos, como el Fe y Mn. En esta práctica parte de la neutralización con cal, se precipita como hidróxidos férricos (III) e hidróxidos de manganeso (IV) se debe controlar el pH para cada etapa. Aunque el pH al comienzo empezó en 2.2, Se ha logrado un efluente de acuerdo con los estándares establecidos para su vertimiento o reutilización. Los hidróxidos secos pueden alcanzar niveles muy rentables, haciendo sostenible el tratamiento y además despejando de metales a los lodos que comúnmente van a la relavera.
\end{abstract}

Palabras clave: Drenaje acido; separación; manganeso; hierro.

\begin{abstract}
Acid effluents from the leaching of sulphide minerals could be a liability that will return part of the cost of treatment when economic elements such as Fe and $\mathrm{Mn}$ are obtained. In this practical part of the neutralization with lime, it is precipitated as ferric hydroxides (III) and manganese (IV) hydroxides the $\mathrm{pH}$ must be controlled for each stage. Although the $\mathrm{pH}$ initially started at 2.2, an effluent has been achieved according to the standards established for its discharge or reuse. Dry hydroxides can reach very profitable levels, making the treatment sustainable and also clearing metals from the sludge that commonly goes to the tailings.
\end{abstract}

Keywords: Acid drainage; separation; manganese; iron.

\footnotetext{
Docente Principal en la Universidad Nacional Mayor de San Marcos. Lima, Perú.

E-mail: icalderond2@unmsm.edu.pe- ORCID: https://orcid.org/0000-0002-1374-9307

2 Universidad Nacional Mayor de San Marcos, Unidad de Posgrado de Geología de Postgrado. Lima, Perú. E-mail: mactuiro@hotmail.com
} 


\section{INTRODUCCIÓN}

Aunque los drenajes ácidos han sido comprobados, recién desde el siglo pasado y es cierto que está asociado a la proliferación e incremento de la minera, estos existen de manera natural desde hace millones de años debido a la transformación en la naturaleza, durante el cual han aparecido aguas acidas a través de las rocas y se han equilibrado con las calizas y otras rocas básicas naturalmente en grandes corrientes de agua. Siendo el resultado en la naturaleza grandes extensiones de suelos con alta contenidos de algunos metales contaminantes. Si observamos el cambio de los mares en el tiempo geológico. Un ejemplo son los yacimientos de óxidos de hierro que se formaron al aumentar el valor de $\mathrm{pH}$ en los océanos antiguos.

La influencia del agua y oxígeno sobre el sulfuro de hierro (FeS2), generan cambios geoquímicos de hidrólisis y oxidación que llevan a la solubilización de los minerales produciéndose aguas ácidas con $\mathrm{pH}$ entre 1,5 y 6 , con muchos sulfatos y metales en solución (Akcil \& Koldas, 2006) como hierro, manganeso, zinc, plomo, cobre, cadmio, arsénico, antimonio entre otros. Llamados drenajes ácidos mineros DAM (Chaparro Leal, 2015). A cantidad de estas aguas son derivadas a canales y emergen al exterior de operaciones subterráneas o son bombeadas hacia los tajos abiertos. Debido a las apreciables cantidades metales y acidez, estas aguas ácidas a fin de evitar su daño ambiental se neutralización totalmente de tal forma que permite obtener un agua tratada y un lodo que contiene óxidos, hidróxidos y carbonatos metálicos (Bamforth et al., 2006), yeso y otras sales (INAP, 2014) como jarosita $\left[\mathrm{KFe}_{3}(\mathrm{OH})_{6}(\mathrm{SO} 4)_{2}\right]$, schwertmannite $\left[\mathrm{Fe}_{8} \mathrm{O}_{8}(\mathrm{OH})_{6} \mathrm{SO}_{4}\right]$, goethite $(\mathrm{a}-\mathrm{FeOOH})$, ferrihydrite $\left(\mathrm{Fe}_{2} \mathrm{O}_{3} 1.8 \mathrm{H}_{2} \mathrm{O}\right) \mathrm{O}$ magnetita $\left(\mathrm{Fe}_{2} \mathrm{O}_{3}\right)$, conteniendo baja concentraciones de otros metales que ocupan grandes extensiones para su disposición y acumulación hasta su plan de cierre final.

Con el estudio geoquímico en minería (Chaparro Leal, 2015) se pudo caracterizar los efluentes productos de la interacción agua/roca; también drenajes de desmontes y relaves de empresas metalúrgicas que optaron por programas de investigación de fraccionamiento de los metales de dichos efluentes para controlar y conducir a la sostenibilidad los impactos positivos ambientales.
La gestión de los drenajes ácidos caracterizado por las reacciones de oxidación del sulfuro, formación de la acides y oxidación del $\mathrm{Fe}^{2+}$ dadas en las ecuaciones (1), (2), (3) acompañadas al final por actividad bacteriana ${ }^{1}$ cuando estos ya se han generado involucra generalmente un tratamiento de neutralización y precipitación que es un tratamiento activo.

$\mathrm{FeS} 2(\mathrm{~s})+7 / 2 \mathrm{O} 2+\mathrm{H} 2 \mathrm{O} \rightarrow \mathrm{Fe}+2+2 \mathrm{SO} 4-2+2 \mathrm{H}+$

$\mathrm{Fe}+2+1 / 4 \mathrm{O} 2+21 / 2 \mathrm{H} 2 \mathrm{O}<->\mathrm{Fe}(\mathrm{OH}) 3(\mathrm{~s})+2 \mathrm{H}+$

$14 \mathrm{Fe}+3+\mathrm{FeS} 2(\mathrm{~s})+8 \mathrm{H} 2 \mathrm{O} \rightarrow 2 \mathrm{SO} 4-2+15 \mathrm{Fe}+2+16 \mathrm{H}$ $+$

Un tratamiento pasivo solo se utiliza cuando la cantidad de los metales en el DAM es baja y el pH es igual o superior a 4.5 .

Por lo tanto, la Neutralización con cal, agitación y floculante presentada en la Figura 1 es la más utilizada en nuestras unidades mineras en el Perú, donde tenemos gran cantidad de yacimientos metálicos ligados a sulfuros como pirita y arsenopirita no utilizables.

Ya que los subproductos de la neutralización constituyen un gran desafío ambiental para la minería, está surgiendo la opción de modificar este proceso para obtener el fraccionamiento de al menos los elementos económicos de los tóxicos, como es el caso del $\mathrm{Zn}, \mathrm{Fe}, \mathrm{Mn}$ de otros como el As, Cd.

Una gran cantidad de las especies de metales se precipitan, pero la eliminación de iones manganeso de la DAM es notoriamente difícil debido a su compleja química en sistemas acuosos (Bamforth et al., 2006). De hecho, para la precipitación total de manganeso como $\mathrm{Mn}(\mathrm{OH})_{2}$, el $\mathrm{pH}$ debe estar por encima de 8 (Ladeira \& Gonalves, 2008).

La solubilidad Kps establece la condición necesaria para la formación de una fase sólida en un medio acuoso, para lo cual se requiere que el producto de la concentración de los iones Q en solución acuosa sea tal que su valor exceda el valor de la constante del producto de solubilidad Ksp, de modo que $(\mathrm{Ksp} / \mathrm{Q})<1$, y esto controladamente permite un fraccionamiento de los iones en solución pasando a una

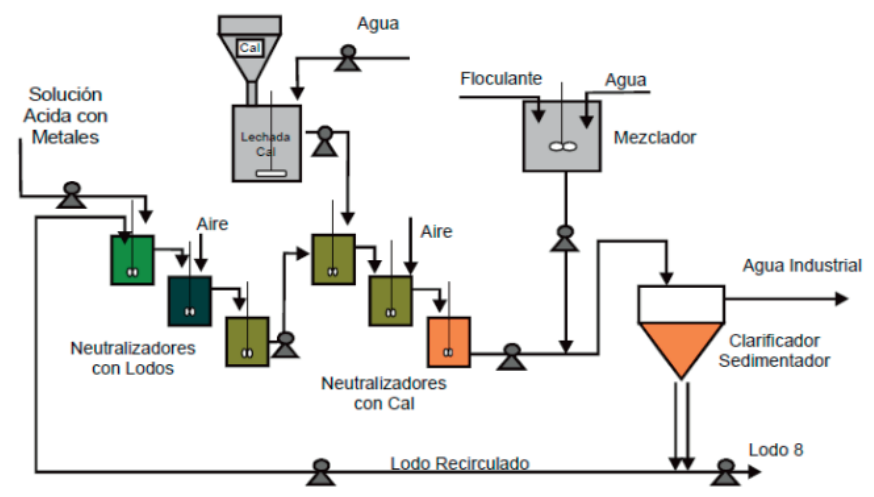

Figura 1. Esquema general de un tratamiento activo de DAM (Chaparro Leal, 2015) 
fase sólida; así tenemos que el $\mathrm{Kps}$ para el $\mathrm{Fe}(\mathrm{OH})_{3}$ es $1.5 \times 10^{-36}$ y del $\mathrm{Mn}(\mathrm{OH})_{2}$ es $2.06 \times 10^{-13}$

La precipitación de los hidróxidos de hierro y manganeso utilizando cal y caliza se indican en las ecuaciones (4), (5) y (6)

$\mathrm{Fe}_{2}(\mathrm{SO} 4)^{3}+3 \mathrm{Ca}(\mathrm{OH}) 2 \rightarrow 2 \mathrm{Fe}(\mathrm{OH})^{3}+3 \mathrm{CaSO} 4$

$\mathrm{Mn}\left(\mathrm{SO}_{4}\right)+\mathrm{Ca}(\mathrm{OH})_{2} \rightarrow \mathrm{Mn}(\mathrm{OH})^{2}+\mathrm{Ca}\left(\mathrm{SO}_{4}\right)$

$\mathrm{Fe}_{2}(\mathrm{SO} 4)^{3}+3 \mathrm{CaCO} 3+3 \mathrm{H} 2 \mathrm{O} \rightarrow 2 \mathrm{Fe}(\mathrm{OH}) 3+3 \mathrm{CaSO} 4+$ $3 \mathrm{CO} 2$
Se utilizan los diagramas de estabilidad de los metales en función del pH (Sicupira et al., 2014) y Eh a fin de realizar procesos de acondicionamiento en determinados rangos para lograr la formación de fases sólidas que permitan su remoción en un proceso de separación sólido/ líquido, que se muestra en la Figura 2 y Figura 3.

La jarosita se puede formar a $\mathrm{pH} \backslash 3$ y alta concentración de sulfatos, mientras que los hidróxidos de hierro y la goetita pueden hacerlo hasta un $\mathrm{pH}$ neutro. La Schwertmannite debe ser precipitada entre ph 3 y 4 . Las estructuras de la jarosita y schwertmannite son conocidas por ser inestables, pero podrían ser transformadas en formas más estables de precipitados de hierro como goetita o magnetita

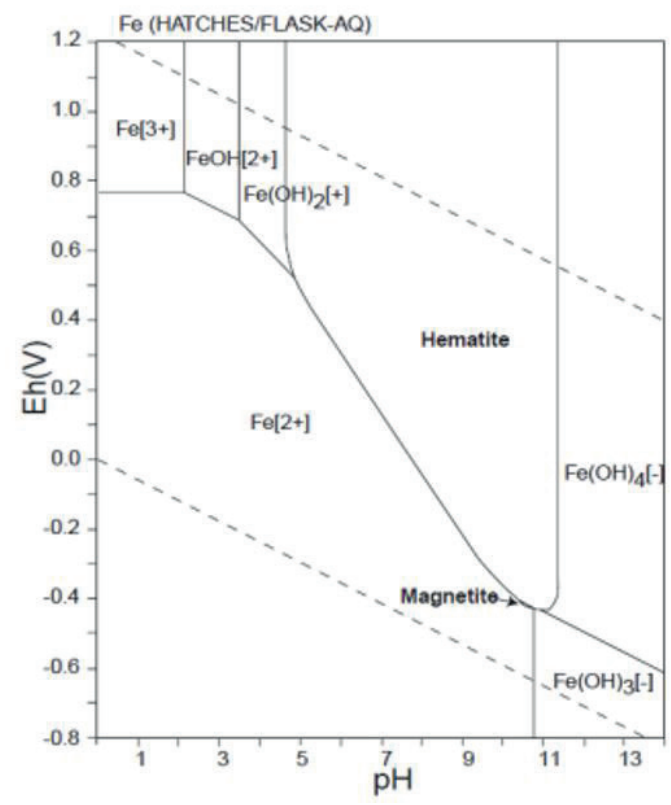

Figura 2. Diagrama de estabilidad de $\mathrm{pH}$ y Eh del hierro

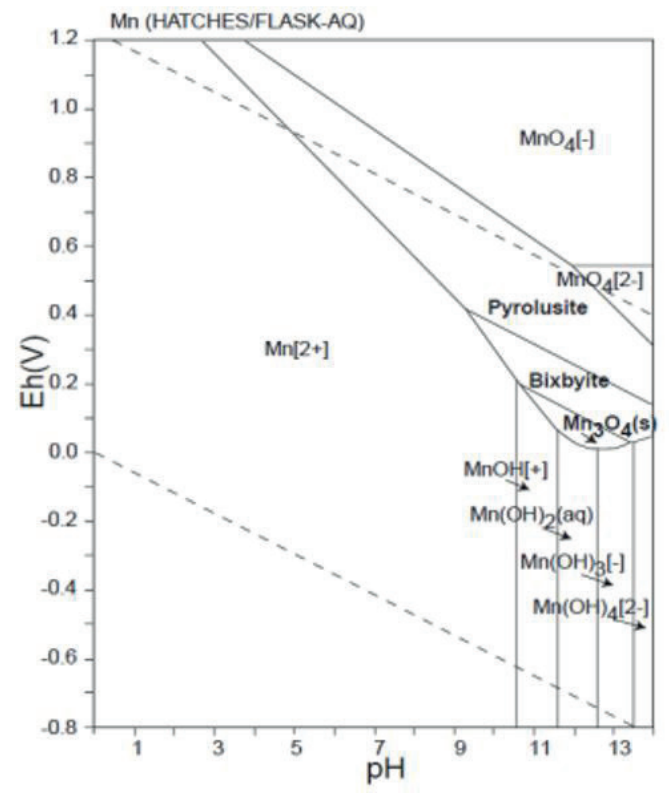

Figura 3. Diagrama de estabilidad de $\mathrm{pH}$ y Eh del manganeso 


\section{MÉTODOS}

\subsection{Parte Experimental}

\subsubsection{Caracterización del efluente}

La secuencia metodológica fue primero caracterizar el efluente del DAM (Watten et al., 2005), por espectrometría de Absorción Atómica.

\subsubsection{Precipitación y separación de los metales.}

Se desarrolla en una primera prueba de precipitación de los iones hierro y manganeso utilizando volúmenes de 2 litros de agua destilada con adición de sulfato férrico y sulfato manganoso grados reactivos en forma independiente de 3000 ppm, agitados a 250 RPM con controles del pH y potencial redox y adición de solución de lechada de cal para elevar el pH (al $10 \%$ y en agitación) y floculante al final para la separación sólido/líquido, para determinar las características de precipitación.

En una segunda etapa se trabaja con el efluente minero ácido, se le fracciona primero el hierro, se deja decantar y se trasvasa la solución que contiene al manganeso en solución a otro vaso. Luego, el manganeso es precipitado bajo el mismo procedimiento, con obtención final de un agua tratada y 2 lodos por separado de Hierro y manganeso

Se caracteriza los valores de hierro y manganeso y los demás metales del efluente resultante por espectrometría de absorción atómica (ver Figura 4 y Figura 5).

\section{RESULTADOS Y DISCUSIÓN}

\subsection{Caracterización del efluente}

Para su caracterización se analizaron los parámetros mostrados en la Tabla 1

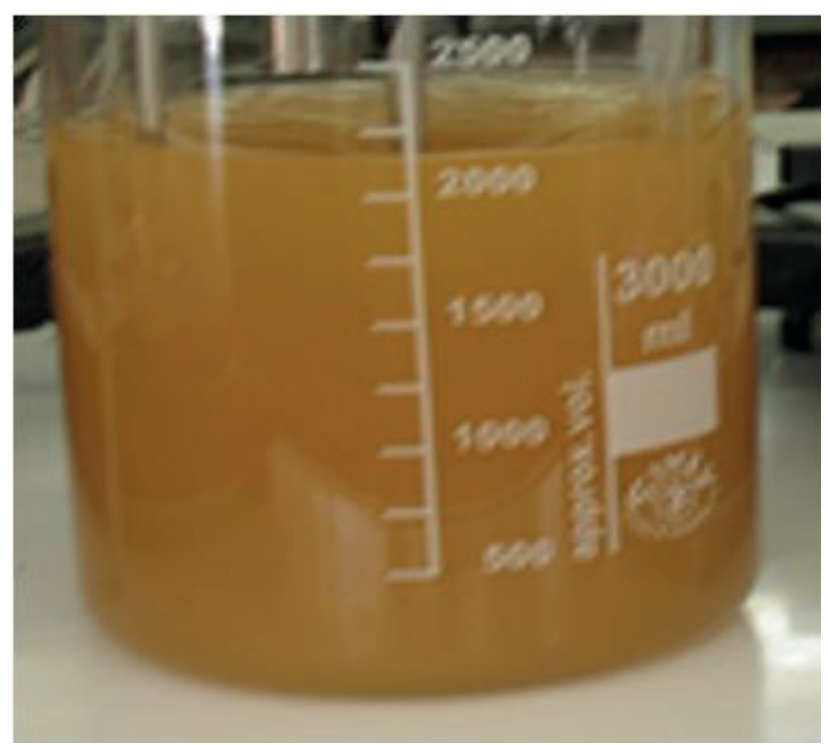

Figura 4. Efluente acido de $\mathrm{pH} 2.2$

(Elaboración propia)
Tabla 1. Características del efluente DAM

\begin{tabular}{ccc}
\hline Parámetro & Unidad & Valores \\
\hline $\mathrm{pH}$ & - & 2.2 \\
Sulfatos & $\mathrm{mg} / \mathrm{L}$ & 3140 \\
$\mathrm{Fe}$ & $\mathrm{mg} / \mathrm{L}$ & 2815 \\
$\mathrm{Mn}$ & $\mathrm{mg} / \mathrm{L}$ & 897 \\
$\mathrm{Cu}$ & $\mathrm{mg} / \mathrm{L}$ & 8.076 \\
$\mathrm{~Pb}$ & $\mathrm{mg} / \mathrm{L}$ & 2.372 \\
$\mathrm{Zn}$ & $\mathrm{mg} / \mathrm{L}$ & 3.111 \\
$\mathrm{Cd}$ & $\mathrm{mg} / \mathrm{L}$ & 0.031 \\
$\mathrm{As}$ & $\mathrm{mg} / \mathrm{L}$ & 0.172 \\
Conductividad & $\mu \mathrm{S} / \mathrm{cm}$ & 4630 \\
\hline
\end{tabular}

\subsection{Prueba de caracterización de las condiciones}

Se obtienen los $\mathrm{pH}$ de precipitación del Fe y del Mn de acuerdo con las figuras 6 y 7

\subsubsection{Primera etapa- precipitación de Fe del efluente}

Se muestran los resultados de los ensayos de la prueba de precipitación del Fe hasta persistencia de $\mathrm{pH} 3$ y subida hasta pH 5.5 (ver Tabla 2).

Tabla 2. Valores para la precipitación del Fe del efluente DAM

\begin{tabular}{cc}
\hline Vol. Cal (ml) & pH \\
\hline 0 & 2.2 \\
5 & 2.7 \\
10 & 3.2 \\
30 & 3.3 \\
40 & 3.6 \\
50 & 3.8 \\
55 & 4.0 \\
65 & 5 \\
67 & 5.5 \\
\hline
\end{tabular}

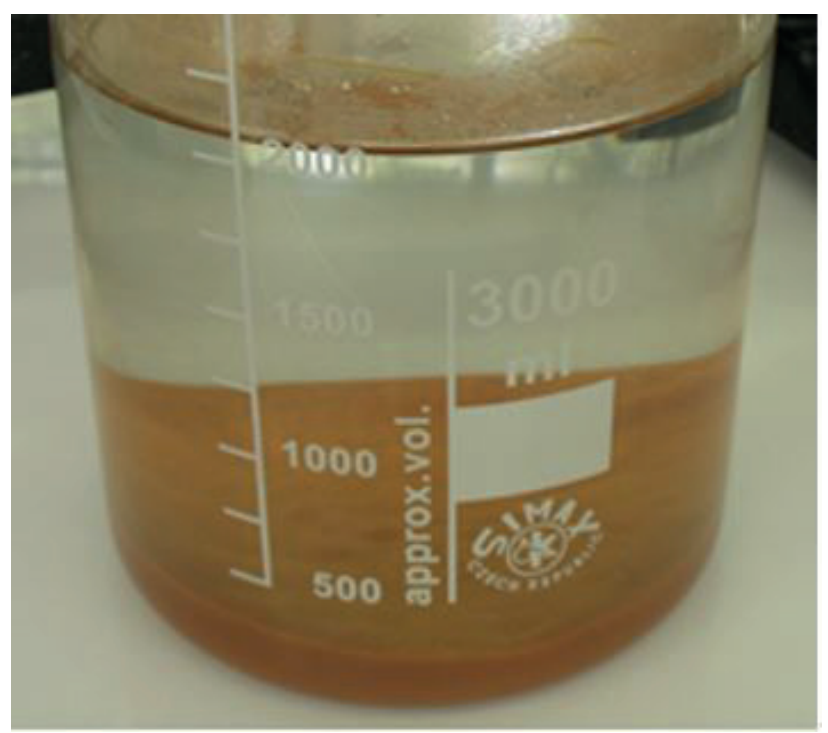

Figura 5. Precipitado de Fe separado del Mn (Elaboración propia) 


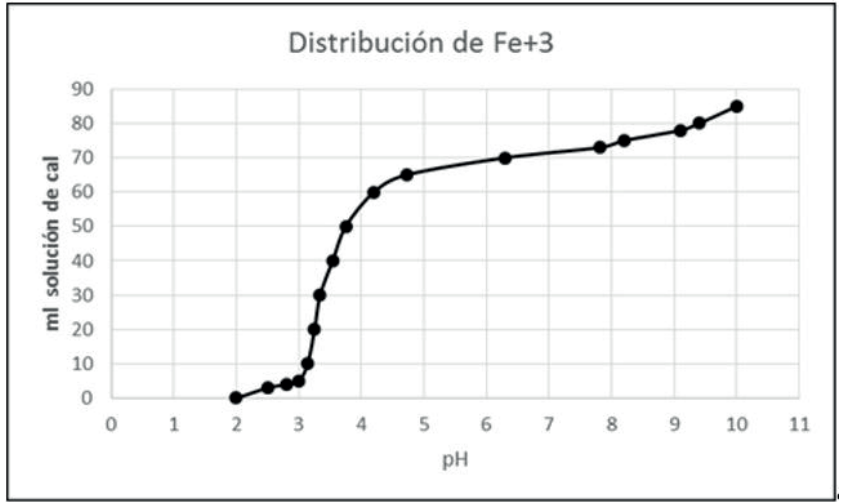

Figura 6. Precipitación del Fe cuantitativamente a partir de $\mathrm{pH} 3$ (Elaboración propia)

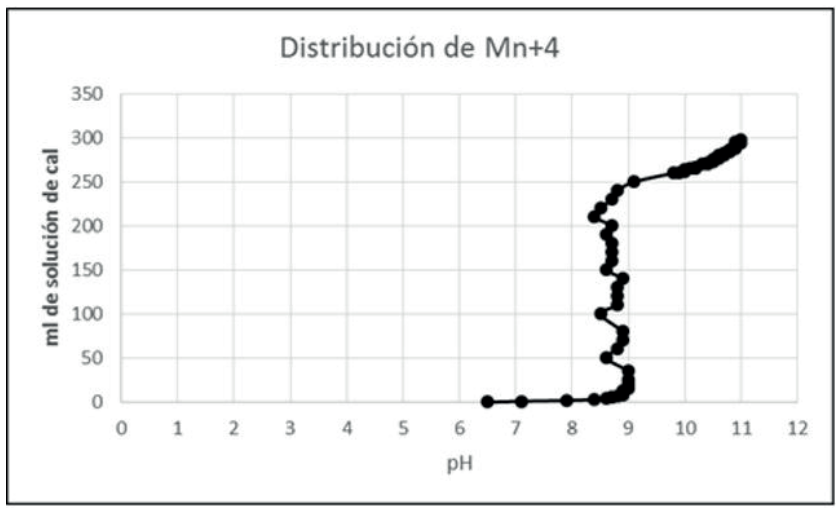

Figura 7. Precipitación del Mn cuantitativamente a partir de pH 8.5

\subsubsection{Segunda etapa- precipitación de Mn del efluente}

Se muestran los resultados de los ensayos de la prueba de precipitación del Mn desde persistencia de $\mathrm{pH} 6.5$ y hasta $\mathrm{pH}$ persistencia de $\mathrm{Ph} 8.5$ y subida hasta $\mathrm{Ph} 9$ (ver Tabla 3 ).

Tabla 3. Valores para la precipitación del Mn del efluente DAM

\begin{tabular}{cc}
\hline Vol. Cal (ml) & $\mathrm{pH}$ \\
\hline 0 & 5.5 \\
5 & 7 \\
10 & 8.5 \\
20 & 8.7 \\
50 & 8.8 \\
65 & 8.9 \\
72 & 9.0 \\
\hline
\end{tabular}

3.3. Caracterización del efluente residual después de la separación de metales (Tabla 4).

Tabla 4. Características del efluente DAM

\begin{tabular}{ccc}
\hline Parametro & Unidad & Valores \\
\hline $\mathrm{pH}$ & - & 8.9 \\
Sulfatos & $\mathrm{mg} / \mathrm{L}$ & 220 \\
$\mathrm{Fe}$ & $\mathrm{mg} / \mathrm{L}$ & 0.025 \\
$\mathrm{Mn}$ & $\mathrm{mg} / \mathrm{L}$ & 0.018 \\
$\mathrm{Cu}$ & $\mathrm{mg} / \mathrm{L}$ & 0.001 \\
$\mathrm{~Pb}$ & $\mathrm{mg} / \mathrm{L}$ & 0.001 \\
$\mathrm{Zn}$ & $\mathrm{mg} / \mathrm{L}$ & 0.002 \\
$\mathrm{Cd}$ & $\mathrm{mg} / \mathrm{L}$ & 0.001 \\
$\mathrm{As}$ & $\mathrm{mg} / \mathrm{L}$ & 0.001 \\
\hline
\end{tabular}

\section{CONCLUSIONES}

- El proceso de fraccionamiento de metales de un efluente ácido minero se desarrolla controlando sistemáticamente el $\mathrm{pH}$ del medio acuoso y su oxidación para su paso a sus fases sólidas como hidróxidos.

- La utilización de solución saturada de cal al $10 \%$ permite un buen control en la separación del hierro y manganeso. Logrando mantener los $\mathrm{pH}$ en 5.5 y 9 para cada etapa.

- Podemos observar que la aireación por la agitación permite pasar el $\mathrm{Mn}+2$ a $\mathrm{Mn}+4$; esta cinética podría ser acelerada utilizando $\mathrm{H}_{2} \mathrm{O}_{2}$.

- El agua final tratada cumple con las condiciones para su descarga en cuerpos receptores de acuerdo con el ECA.

- Los valores de hierro y manganeso obtenidos al final del proceso corresponden a recuperaciones del $92 \%$ y $90 \%$ necesarios para uso industrial.

\section{AGRADECIMIENTOS}

El agradecimiento a la Universidad Nacional Mayor de San Marcos y al Instituto de Investigación, que permitió desarrollar y publicar el presente artículo. 


\section{REFERENCIAS}

Akcil, A., \& Koldas, S. (2006). Acid Mine Drainage (AMD): causes, treatment and case studies. In Journal of Cleaner Production (Vol. 14, Issues 12-13 SPEC. ISS., pp. 11391145). https://doi.org/10.1016/j.jclepro.2004.09.006

Bamforth, S. M., Manning, D. A. C., Singleton, I., Younger, P. L., \& Johnson, K. L. (2006). Manganese removal from mine waters - investigating the occurrence and importance of manganese carbonates. Applied Geochemistry, 21(8), 12741287. https://doi.org/10.1016/j.apgeochem.2006.06.004

Chaparro Leal, L. T. (2015). Drenajes ácidos de mina formación y manejo. Revista ESAICA, 1(1), 53. https://doi. org/10.15649/24225126.272

INAP. (2014). The Global Acid Rock Drainage GuideGARD Guide. The International Network for Acid Prevention. http://www.gardguide.com/images/5/5f/ TheGlobalAcidRockDrainageGuide.pdf
Ladeira, A. C. Q., \& Gonalves, C. R. (2008). Uranium recovery and manganese removal from acid mine drainage. WIT Transactions on Ecology and the Environment, 111, 465474. https://doi.org/10.2495/WP080451

Sicupira, D. C., Tolentino Silva, T., Leão, V. A., \& Mansur, M. B. (2014). Batch removal of manganese from acid mine drainage using bone char. Brazilian Journal of Chemical Engineering, 31(1), 195-204. https://doi.org/10.1590/ S0104-66322014000100018

Watten, B. J., Sibrell, P. L., \& Schwartz, M. F. (2005). Acid neutralization within limestone sand reactors receiving coal mine drainage. Environmental Pollution, 137(2), 295-304. https://doi.org/10.1016/j.envpol.2005.01.026 